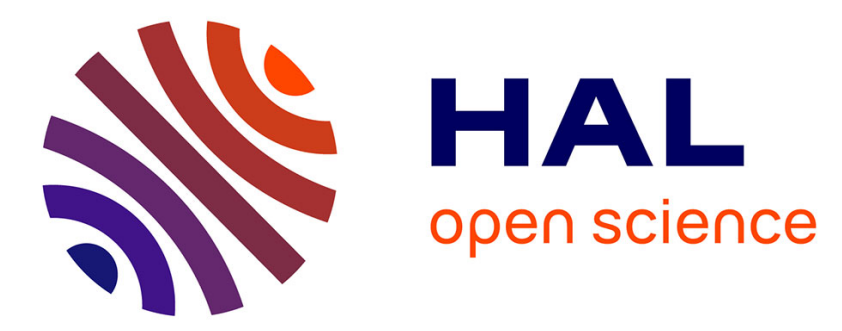

\title{
Near equilibrium mobility tensor in a semiconductor superlattice: scattering by acoustical phonons
}

\author{
J.F. Palmier, Y. Ballini
}

\section{To cite this version:}

J.F. Palmier, Y. Ballini. Near equilibrium mobility tensor in a semiconductor superlattice: scattering by acoustical phonons. Journal de Physique Lettres, 1980, 41 (22), pp.539-542. 10.1051/jphyslet:019800041022053900 . jpa-00231841

HAL Id: jpa-00231841

https://hal.science/jpa-00231841

Submitted on 1 Jan 1980

HAL is a multi-disciplinary open access archive for the deposit and dissemination of scientific research documents, whether they are published or not. The documents may come from teaching and research institutions in France or abroad, or from public or private research centers.
L'archive ouverte pluridisciplinaire HAL, est destinée au dépôt et à la diffusion de documents scientifiques de niveau recherche, publiés ou non, émanant des établissements d'enseignement et de recherche français ou étrangers, des laboratoires publics ou privés. 


\title{
Near equilibrium mobility tensor in a semiconductor superlattice : scattering by acoustical phonons
}

\author{
J. F. Palmier and Y. Ballini \\ Centre National d'Etude des Télécommunications, (LAB/ICM/MPA), BP 40, 22301 Lannion, France
}

(Reçu le 12 juin 1980, révisé le 22 août, accepté le 26 septembre 1980)

\begin{abstract}
Résumé. - On étudie la mobilité à faible champ dans un super-réseau à base de semiconducteurs. Dans le cas de la diffusion par les phonons acoustiques, on obtient des formules analytiques. La discussion des résultats montre que la règle de conservation des $\mathbf{k}$ doit être entourée de précautions dans un tel système (processus Umklapp).
\end{abstract}

\begin{abstract}
The near equilibrium mobility tensor in a one-dimensional superlattice is studied. Analytical formulae are given for acoustical phonon scattering. Discussion of the results shows that the $\mathbf{k}$ conservation rule must be carefully handled (U-processes).
\end{abstract}

Molecular beam epitaxy and, to some extent, other epitaxial techniques permit the construction of multilayered materials called superlattices (S. L.) or multiple quantum wells (M. Q. W.), according to the periodicity dimension [1]. These materials have interesting applications in electronics [2] or optics [3]. In the present letter we deal with S. L. in which transport along the growth direction $z$ is still possible; it implies a partially filled first miniband of reasonable width, supporting transport in the $z$ direction. As for example, if we choose the $\mathrm{Ga}_{1-x} \mathrm{Al}_{x} \mathrm{As} / \mathrm{GaAs}$ system, the critical value of the periodicity $d$ along $z$ is of the order of $50-80 \AA$, according to the $x$ values and the relative width of each material. For higher $d$ values one obtains M. Q. W. in which only transport perpendicular to $z$ is possible, and has been derived on the basis of quantum sized wave functions [4-5]. The present study must include the M. Q. W. in the limit of vanishing bandwith. We also restrict the study to the low field mobility tensor and, for brevity, to the scattering by acoustical phonons ; this case is also interesting as it provides analytical expressions for the mobility tensor. In the case of impurity scattering and polar optical phonon scattering, computer solutions only are possible even with a very simple band model. A chief feature of the S. L. systems is their large anisotropy. Recent pseudopotential calculations [6] show that for very small $d$ values there is practically no difference between the S. L. band structure and that of the corresponding alloy. However, as the M. Q. W., on an other hand, have a very anisotropic behaviour in which electrons are confined in quantum wells, S. L. systems must exhibit all the intermediate cases between tri- and bi-dimensional systems. So, we shall give a mobility tensor of the appropriate symmetry tending to a scalar at the M. Q. W. limit. Numerical results in that limit are quite surprising, showing that something may have been omitted; thus, we shall discuss first principles commonly used in transport calculations. In semiconductors systems one uses the $\mathbf{k}$ vector selection rule in electron-phonon scattering events; this is questionable as phonons of $~ z z$ " momenta larger than $2 \pi / d$ may be important in collisions (in the reduced zone scheme); this is not different from so called U-processes. The relative importance of such events in S. L. systems will be discussed in our last paragraph.

The most widely used model in the superlattice studies is the Kronig-Penney model [7]. It deals with Bloch electrons (or holes) near band extrema of the constitutive materials, and is valid in a range of $d$ values for which a mean potential approximation applies; if $d$ is lower than $10 \AA$, more accurate band theories are required [8], [6]. A weakness of the onedimensional Kronig-Penney model is the lack of knowledge of the electronic behaviour perpendicular to $z$. The reasonable approximation of a transverse average effective mass $m^{*}$ will be used throughout the present paper. Hence the energy $\varepsilon$ versus $\mathbf{k}$ relation :

$$
\varepsilon(\mathbf{k})=\varepsilon_{\perp}+\varepsilon_{\|}=\frac{\hbar^{2} k_{\perp}^{2}}{2 m^{*}}+\Delta\left[1-\cos \left(k_{\|} d\right)\right]
$$

in which $\mathbf{k}_{\perp}, \mathbf{k}_{\|}$are, respectively, the components of $\mathbf{k}$ perpendicular and parallel to the superlattice direction, 
and $\Delta$ is half of the minibandwidth. The $\varepsilon_{\|}$versus $k_{\|}$ variation is given by a phenomenological relation, the exact dependence being more complex $\left(k_{\|}\right.$is restricted to $[-\pi / d, \pi / d])$.

The basic equation which describes electron transport near equilibrium (weak electric field) is the Boltzmann transport equation whose standard solution is :

$$
f(\mathbf{k})=\bar{f}(\varepsilon)+\frac{e \mathbf{E}}{\hbar} \cdot \nabla_{k} \bar{f} \cdot \tau(\mathbf{k})
$$

in which $\bar{f}(\varepsilon)$ is the equilibrium distribution function, $e$ the electron charge, $\mathbf{E}$ the electric field, and $\tau(\mathbf{k})$ the collision time given by :

$$
\tau(\mathbf{k})=\left[\sum_{\mathbf{k}^{\prime}} W\left(\mathbf{k} \rightarrow \mathbf{k}^{\prime}\right)\right]^{-1}
$$

$W\left(\mathbf{k} \rightarrow \mathbf{k}^{\prime}\right)$ being elemental transition probability per unit time. Electron-phonon scattering processes lead to the classical form of $W$ :

$$
\begin{aligned}
W_{\mathrm{e}-\mathrm{p}}^{ \pm}=\frac{2 \pi}{\hbar} \mid\left\langle\mathbf{k}^{\prime}\right| H_{1}^{ \pm} & \left.|\mathbf{k}\rangle\right|^{2} \times \\
& \times\left(N_{q}+\frac{1}{2} \pm \frac{1}{2}\right) \delta\left(U^{ \pm}\right)
\end{aligned}
$$

in which $( \pm)$ sign is used to outline the difference between phonon $q$ emission and absorption. The important features of (4) are classically the energy and momentum conservation rules :

$$
\begin{gathered}
U^{ \pm}=\frac{\hbar^{2}}{2 m^{*}}\left(k_{\perp}^{\prime 2}-k_{\perp}^{2}\right)+\Delta\left(\cos k_{\|} d-\cos k_{\|}^{\prime} d\right) \pm \\
\pm \hbar \omega(q) \\
\mathbf{k}^{\prime}=\mathbf{k} \pm \mathbf{q} .
\end{gathered}
$$

The summation over $k^{\prime}$ in (3) can be replaced by an integration in cylindrical coordinates $\left(k_{\perp}^{\prime}, k_{\|}^{\prime}, \theta^{\prime}\right)$. The first step of that integration is made with the help of change of variables $U^{ \pm} \leftrightarrow k^{\prime}$. The associated Jacobians are easy to find from (5) and (6), namely if collisions are considered as quasi-elastic :

$$
\frac{\partial U^{ \pm}}{\partial k_{\perp}^{\prime}} \simeq \frac{\hbar^{2}}{m^{*}} k_{\perp}^{\prime}
$$

The second and third steps are not difficult to perform except for the condition that $U^{ \pm}$actually crosses the points $U^{ \pm}=0$. For acoustical phonons with (7) the integration over $\theta$ only gives a factor $2 \pi$, and integration over $k_{\|}^{\prime}$ is restricted to domains $\Gamma^{+}$ and $\Gamma^{-}$. The restrictions $\left(\Gamma^{ \pm}\right)$of $k_{\|}^{\prime}$ correspond to the simultaneous conditions $U^{ \pm}=0$ and $k_{\perp}^{\prime}>0$. Domains $\Gamma^{+}$and $\Gamma^{-}$are equivalent for quasi-elastic collisions. At high temperatures the equilibrium phonon average occupation numbers are close to $k_{\mathrm{B}} T / \hbar q s$ and we obtain for $\tau$ :

$$
\begin{gathered}
\frac{1}{\tau_{\mathrm{a}}}=\frac{1}{\rho \pi} E_{1}^{2} \frac{m^{*}}{s^{2} \hbar^{3}} k_{\mathrm{B}} T \cdot k_{1}\left(k_{\perp}, k_{\|}\right) \\
k_{1} d=\left\{\begin{array}{lll}
\operatorname{Arc} \cos \left(\cos k_{\|} d-\varepsilon_{\perp} / \Delta\right) & \text { if } & \mathbf{k} \in(\mathrm{D}) \\
\pi & \text { if } & \mathbf{k} \in\left(\mathrm{D}^{\prime}\right)
\end{array}\right.
\end{gathered}
$$

in (8) $E_{1}, \rho, s$ are respectively the deformation potential, the density of the crystal, the sound velocity. The physical meanings of $(\Gamma)$, (D) and $\left(\mathrm{D}^{\prime}\right)$ are commented upon in the caption for figure $1 .(\Gamma)$ is the domain of integration over $k_{\|}^{\prime}$ and is smaller than $[-\pi / d, \pi / d]$ if

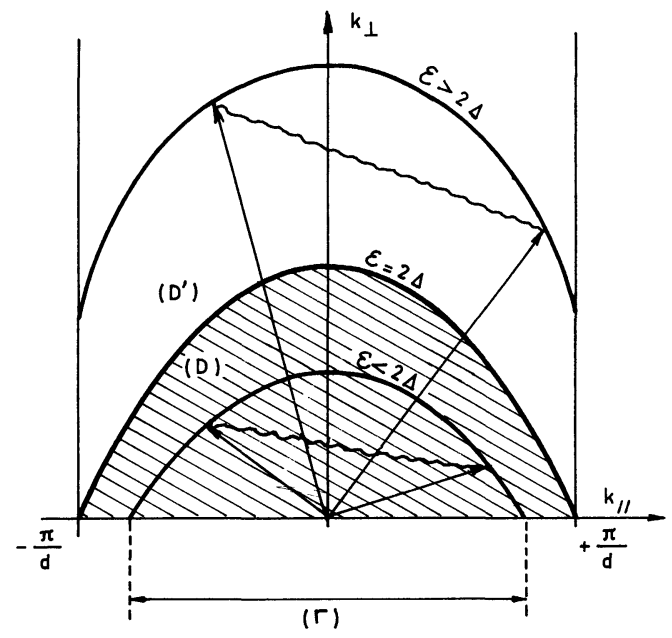

Fig. 1. - Representation of constant-energy lines $\varepsilon(\mathbf{k})=$ const. in the plane $\left(k_{1}, k_{\|}\right)$. The domain D is limited by $\varepsilon(k)=2 \Delta$. Inside $\mathrm{D}$ there is a limiting zone $(\Gamma)$ for $k_{\|}^{\prime}$ component, whereas for the domain $\mathrm{D}^{\prime}$ outside $\mathrm{D}$, all the $k_{\|}^{\prime}$ values in $[-\pi / d, \pi / d]$ are allowed.

the corresponding constant energy line crosses the axis $k=0$. Hence a critical domain (D) in the $\left(k_{\perp}, k_{\|}\right)$ plane can be defined. Outside (D) there is always some $k_{1}$ which obeys the relations (5) and (6) whereas inside (D) a couple $\left(k_{\perp}^{\prime}, k_{\|}^{\prime}\right)$ may exist or not according to the limit $(\Gamma)$ at $k_{\perp}^{\prime}=0$. The bi-dimensional case $(\Delta=0)$ is easily found as the limit of (D) vanishing : $k_{1} d=\pi$ and $\tau_{\perp}$ is independent on the energy.

Now we come to transport parameters. In order to simplify, we consider thermal averages with a Maxwell equilibrium distribution. Then the mobility tensor can be derived from (2), (8) :

$$
\overline{\overline{\boldsymbol{\mu}}}=\left(\begin{array}{ccc}
\mu_{\perp} & 0 & 0 \\
0 & \mu_{\perp} & .0 \\
0 & 0 & \mu_{\|}
\end{array}\right) .
$$

The form (10) for the mobility tensor is generally true for (100) superlattices grown on zinc blende sublattices, resulting in a quadratic $\mathrm{D}_{2 \mathrm{~d}}$ point group. It is important to derive $\overline{\bar{\mu}}$ with respect to the $3 D$ 
corresponding mobility (with the same average value $\left.m^{*}\right)$. A classical derivation gives :

$$
\begin{aligned}
\frac{\mu_{\perp}}{\mu_{3 \mathrm{D}}}= & \frac{3}{\sqrt{8 \pi}}=\sqrt{\frac{k_{\mathrm{B}} T m^{*} d^{2}}{\hbar^{2}}} \times \\
& \times\left[\left\langle u \frac{\pi}{k_{1} d}\right\rangle_{\mathrm{D}}+\langle u\rangle_{\mathrm{D}^{\prime}}\right] \\
\frac{\mu_{\|}}{\mu_{3 \mathrm{D}}}= & \frac{3}{\sqrt{8 \pi}}\left(\frac{m^{*}}{M}\right)^{3 / 2}\left(\frac{\Delta}{k_{\mathrm{B}} T}\right)^{1 / 2} \times \\
& \times\left[\left\langle\left(\sin ^{2} k_{\|} d\right) \frac{\pi}{k_{1} d}\right\rangle_{\mathrm{D}}+\left\langle\sin ^{2} k_{\|} d\right\rangle_{\mathrm{D}^{\prime}}\right]
\end{aligned}
$$

$M$ is the effective mass at the origin of the miniband and thermal averages $\langle>$ are made in the plane $\left(u=\varepsilon_{\perp} / k_{\mathrm{B}} T, k_{\|}\right)$. The bracket terms in (11) and (12) tend respectively to 1 and $1 / 2$ in the $2 \mathrm{D}$ case.

The limits of (11) and (12) when $\Delta \rightarrow 0$ are in agreement with results reported in [5] (formula (18) of ref. [5]). However, the numerical value of $\mu_{\perp}$ is surprisingly high : at $T=300 \mathrm{~K}$, with $m^{*}=0.1 m_{0}$, $d=50 \AA$ one obtains $\mu_{\perp}=\mu_{3 \mathrm{D}}$ and $\mu_{\perp}$ becomes higher at higher $d$ values. As electrons cannot escape electron-phonon scattering events, one must look more precisely at the basic mechanisms. We feel the main problem occurs in passing from (4) to (6) : the quasi-momentum conservation rule must be handled carefully in such systems. Umklapp processes [9] have been previously involved in Raman scattering studies [10], the exact interpretation of the date being a matter of controversy [11].

In the reduced zone scheme, the electrons' $z$ components of momenta are in $[-\pi / d,+\pi / d]$, but phonons of momenta higher than $2 \pi / d$ could cause collisions if (6) were ruled out. In the present paragraph, we discuss of that possibility. Generally speaking, U-processes occur if the short-range oscillating part of electron wave functions involves more than one crystal plane wave. In usual semiconductors this is always the case, and does not bear any more complexity if there are some equivalent valleys. To be consistent with the chosen Kronig-Penney (K.P.) model of S.L. band structure we must use the corresponding wave

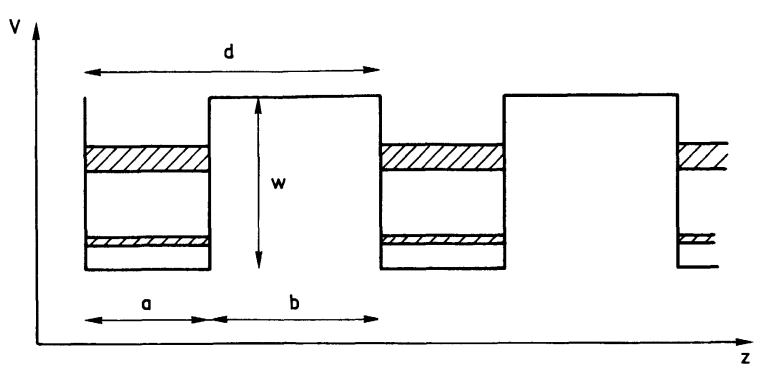

Fig. 2. - Model potential used in the derivation of $V(G)$. functions to obtain selection rules. To be more simple, we shall only give a proof based upon a perturbation analysis. Bloch electrons are supposed to be subjected to a mean potential arising from the difference between electron affinities in both materials (see Fig. 2). That superlattice potential is a function of $z$ only we can expand in Fourier series :

$$
V(z)=\sum_{G} V(G) \mathrm{e}^{i G z}
$$

with parameters given in figure 2 :

$$
V(G)=\frac{b}{d} W \frac{\sin (G b / 2)}{G b / 2} .
$$

The effective mass and the centre zone approximation being assumed for unperturbed energy wave functions, first-order perturbation theory gives :

$$
\psi^{(1)}(\mathbf{k})=|\mathbf{k}\rangle+\sum_{G} V(G) \frac{\left|k_{\|}+G\right\rangle}{\varepsilon\left(k_{\|}\right)-\varepsilon\left(k_{\|}+G\right)}
$$

in which $\left|k_{\|}+G\right\rangle$ is the unperturbed Bloch function whose $k_{\|}$component has been translated of $G(G=2 N \pi / d, N$ integer $)$. In fact, from the K.P. wave functions, we expect much more perturbed states than given by (15), as the K.P. model mixes propagating and evanescent states in the energy area interesting for transport (see shaded area in Fig. (2)). However, the series (15) are interesting in the sense they are direct expansions on $G$ values. Now, we can evaluate matrix element entering (4); the relative intensity of the first U-process $(|G|=2 \pi / d)$ to the N-process $(G=0)$ is, for matrix elements :

$$
\frac{\left|\left\langle\psi^{(1)}\left|H_{1}\right| \psi^{(1)}\right\rangle\right|^{2}}{\left|\left\langle\psi^{(0)}\left|H_{1}\right| \psi^{(0)}\right\rangle\right|^{2}}=1+\frac{4}{\pi^{2}} \frac{W^{2}}{(\varepsilon(G))^{2}}
$$

in which the following simplifications have been made : $\mathbf{k}^{\prime}=\mathbf{k}=0, d=2 a$. Applying the same numerical assumption as previously, equation (16) shows that $U$-processes contribute to $\sim 50 \%$ of scattering for $d=50 \AA$. We are aware that our estimate is very rough, and probably underestimates the intensity of U-processes with respect to the more accurate K.P. wave functions.

We have given a general derivation of the mobility tensor in a semiconductor superlattice; the acoustical phonon scattering leads to analytical results. Discussion of the results has shown the necessity to handle carefully electron-phonon processes. Generalization of this work to inelastic processes (e.g. polar optical phonons) will be published in a more detailed forthcoming paper. 


\section{References}

[1] Review paper : Chang, L. L. and Esaki, L., Prog. Crystal Growth Charact. 2 (1979) 3-14.

[2] Dingle, R., Stormer, H. L., Gossard, A. C., Wiegmann, W., Appl. Phys. Lett. 33 (1978) 665.

[3] Tsang, W. T., Weisbuch, C., Miller, R. C., Dingle, R., Appl. Phys. Lett. 35 (1979) 673.

[4] Mori, S. and Ando, T., J. Phys. Soc. Japan 48 (1980) 865.

[5] Hess, K., Appl. Phys. Lett. 35 (1979) 484.

[6] Andreoni, W., Car, R., Phys. Rev. B. 21 (1980) 3334.

[7] Esaki, L. and Chang, L. L., Phys. Rev. Lett. 33 (1974) 495 Lebwohl, P. and Tsu, R., J. Appl. Phys. 41 (1970) 2664. and Muknerji, D., Nag, B. R., Phys. Rev. B. 12 (1975) 4338.
[8] Caruthers, E., Lin Chung, P. J., Phys. Rev. B 17 (1978) 2705.

[9] Ziman, J. M., Electrons and Phonons (Oxford Univ. Press, London) 1962.

[10] Sai-Halasz, G. A., Pinczuk, A., Yu, P. Y., Esaki, L., Solid State Commun. 25 (1978) 381.

[11] Merlin, R., Colvard, C., Klein, M. V., Morkoc, H., Cho, A. Y, Gossard, A. C., Appl. Phys. Lett. 36 (1980) 43.

[12] Review paper : Dingle, R., Festkorperprobleme $X V$ (Pergamon-Vieweg, Berlin) 1975. 